\title{
Retraction Note to: Postconditioning promotes the cardiac repair through balancing collagen degradation and synthesis after myocardial infarction in rats
}

\author{
Zhang-Feng Wang $\cdot$ Ning-Ping Wang $\cdot$ \\ Suzanna Harmouche · Tiji Philip • Xue-Fen Pang • \\ Feng Bai $\cdot$ Zhi-Qing Zhao
}

Published online: 12 February 2015

(C) Springer-Verlag Berlin Heidelberg 2015

\section{Retraction to: Basic Res Cardiol (2013) 108:318 \\ DOI 10.1007/s00395-012-0318-9}

The article "Postconditioning promotes the cardiac repair through balancing collagen degradation and synthesis after myocardial infarction in rats", Basic Res Cardiol (2013) $108: 318$, was retracted by the authors who regret to have used different fields of the same samples for MMP staining and western blot assay to represent two distinct groups on two occasions in Figs. 4 and 5. Some of the raw data for the earlier experiments with the use of echocardiography for Figs. 8 and 9a were not available for further analysis to exclude that also the same samples were used for two distinct groups. The authors regret the effect of this action on the work of other investigators.

The online version of the original article can be found under doi:10. 1007/s00395-012-0318-9.

The article "Postconditioning promotes the cardiac repair through balancing collagen degradation and synthesis after myocardial infarction in rats", Basic Res Cardiol (2013) 108:318, was retracted by the authors who regret to have used different fields of the same samples for MMP staining and western blot assay to represent two distinct groups on two occasions in Figs. 4 and 5. Some of the raw data for the earlier experiments with the use of echocardiography for Figs. 8 and 9a were not available for further analysis to exclude that also the same samples were used for two distinct groups. The authors regret the effect of this action on the work of other investigators.

Z.-F. Wang · N.-P. Wang · S. Harmouche $\cdot$ T. Philip ·

Z.-Q. Zhao

Cardiovascular Research Laboratory,

Mercer University School of Medicine, Savannah,

GA, USA

\section{Z.-F. Wang}

Department of Otolaryngology, First Affiliated Hospital of Sun

Yat-Sen University, Guang Zhou, China

X.-F. Pang · F. Bai $\cdot$ Z.-Q. Zhao

Department of Physiology, Shanxi Medical University, Taiyuan, Shanxi, China

\section{Z.-Q. Zhao $(\bowtie)$}

Department of Basic Biomedical Sciences, Mercer University

School of Medicine, 4700 Water Avenue,

Savannah, GA 31404, USA

e-mail: zhao_z@mercer.edu 
